\title{
Giant Thoracic Meningocele Causing Acute Respiratory Compromise
}

\author{
Alp Yurter, Paul E. Kaloostian \\ Johns Hopkins University Medical Center, Baltimore, USA \\ Email: paulkaloostian@hotmail.com
}

Received August 29, 2013; revised September 29, 2013; accepted October 6, 2013

Copyright (C) 2013 Alp Yurter, Paul E. Kaloostian. This is an open access article distributed under the Creative Commons Attribution License, which permits unrestricted use, distribution, and reproduction in any medium, provided the original work is properly cited.

\begin{abstract}
Objective: The authors report a rare case of giant thoracic meningocele causing acute respiratory compromise, treated with a ventriculoperitoneal shunt. Case Report: We report the case of a 36-year-old with severe scoliosis status post repair over a decade ago, neurofibromatosis type I, and a known large meningocele in the left thoracic cavity, presenting with new acute respiratory compromise. She was taken to the operating room for a lumboperitoneal shunt, but the operation was aborted due to her severe spinal deformity. Two days later, she successfully underwent a procedure for ventriculoperitoneal shunt placement. Upon discharge a week later, the patient was hemodynamically stable, able to move all extremities with good strength, and demonstrated improved oxygenation. In the following 7 months, the patient demonstrated continued minimal requirement on nasal cannula, and MRI showed a stable left thoracic giant meningocele. Conclusion: Ventriculoperitoneal shunting is a method of treating and stabilizing acutely symptomatic giant meningoceles.
\end{abstract}

Keywords: Giant Thoracic Meningocele; Intrathoracic; Cerebrospinal Fluid (CSF); Ventriculoperitoneal (VP) Shunt; Respiratory Compromise; Neurofibromatosis Type I (NF-I)

\section{Introduction}

A spinal meningocele is a CSF-filled sac resulting from herniation of the meninges through dilated intervertebral foramina or bone defects. Intrathoracic meningoceles are located in the posterior mediastinum and are associated with pathological thoracic vertebrae [1-3]. Though rare, intrathoracic meningoceles are associated with neurofibromatosis type I (NF-I) $60 \%$ - 85\% of the time. They are typically in small or intermediate size. Further, while intrathoracic meningoceles associated with NF-I are usually asymptomatic, patients may present with back pain, radicular pain, headache, cough, and dyspnea [1].

Surgery is typically indicated for symptomatic meningoceles. This process commonly involves removal of the sac via thoracotomy, laminectomy, and costotransversectomy $[1,2,4]$. However, in the presence of respiratory difficulties, surgery under general anesthesia can carry a high risk of mortality [2]. As such, shunts may be used as an alternative to reduce the mass size and CSF pressure [2].

The authors present a rare case of giant thoracic meningocele compromising respiration treated using a ventriculoperitoneal (VP) shunt.

\section{Case Report}

A 36-year-old female with severe scoliosis status post repair over a decade ago, NF-I, and a known large meningocele in the left thoracic cavity presented with new acute respiratory compromise with $\mathrm{O}_{2}$ saturations in the 70's. Recently, her respiratory status had affected her life she had been unable to work. MRI demonstrated a giant left thoracic meningocele emanating from a large dilated neuroforamina at the T3-T4 and/or T4-T5 level (Figures 1 and 2) that was compressing her left lung (Figure 3). She had no neurological deficits despite severe compression of the spinal cord at the T2 level. She was administered face mask oxygen therapy for her dyspnea, as well as a lumbar drain to reduce her CSF pressure.

She was taken to the operating room for an attempted lumboperitoneal shunt. Her existing lumbar drain was removed without issues. However, the procedure was aborted due to severe scoliotic deformity and history of pleural effusion. The patient tolerated the procedure well. She was postoperatively re-intubated for poor respiratory status shortly after extubation, until her respiration stabilized.

Two days later, she was taken back to the operating 


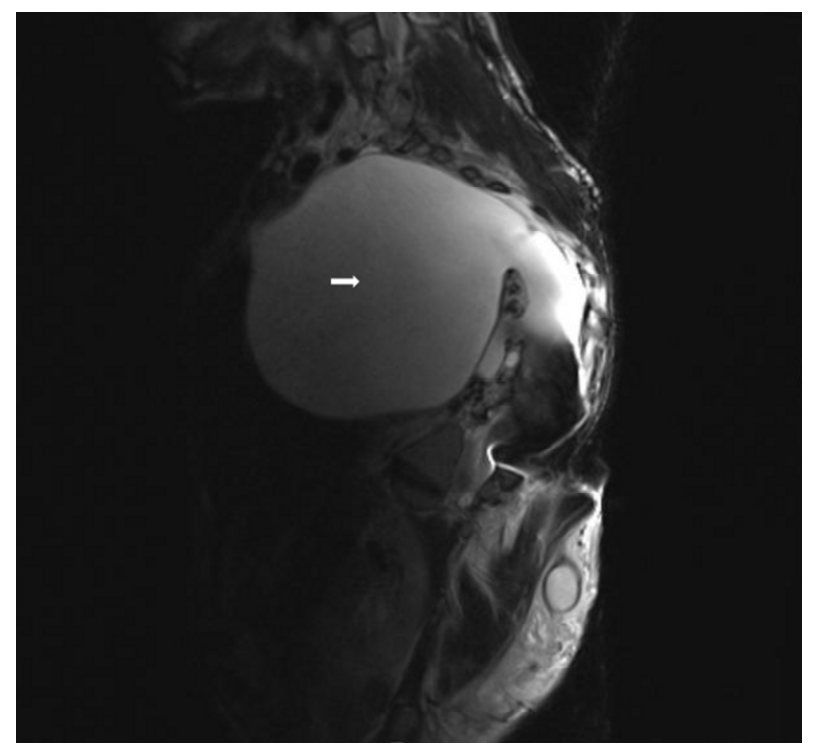

Figure 1. Preoperative sagittal MRI demonstrates giant left thoracic meningocele emanating from a large dilated neuroforamina at the T3-T4 and/or T4-T5 level.

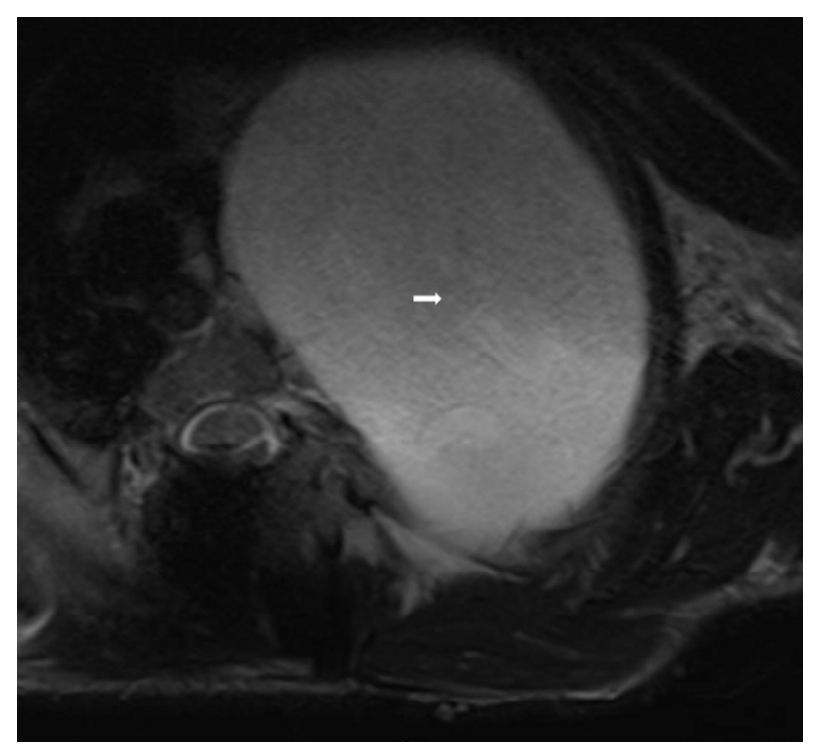

Figure 2. Preoperative axial MRI demonstrates giant left thoracic meningocele emanating from a large dilated neuroforamina at the T3-T4 and/or T4-T5 level.

room for VP shunt placement using an adjustable pressure valve. She underwent the operation and tolerated the procedure well, with no complications. She was brought to the recovery room in stable condition. Her postoperative head CT showed some hemorrhage along the shunt tract, and she got a postoperative shunt survey. Once she was weaned off extubation, she had a post-anesthesia evaluation. She was found to have tachypnea, tachycardia, and mild respiratory distress. Per protocol she received EKG, ABG, chest x-ray, and CT evaluation, as well as pulmonary consult. She was recommended to

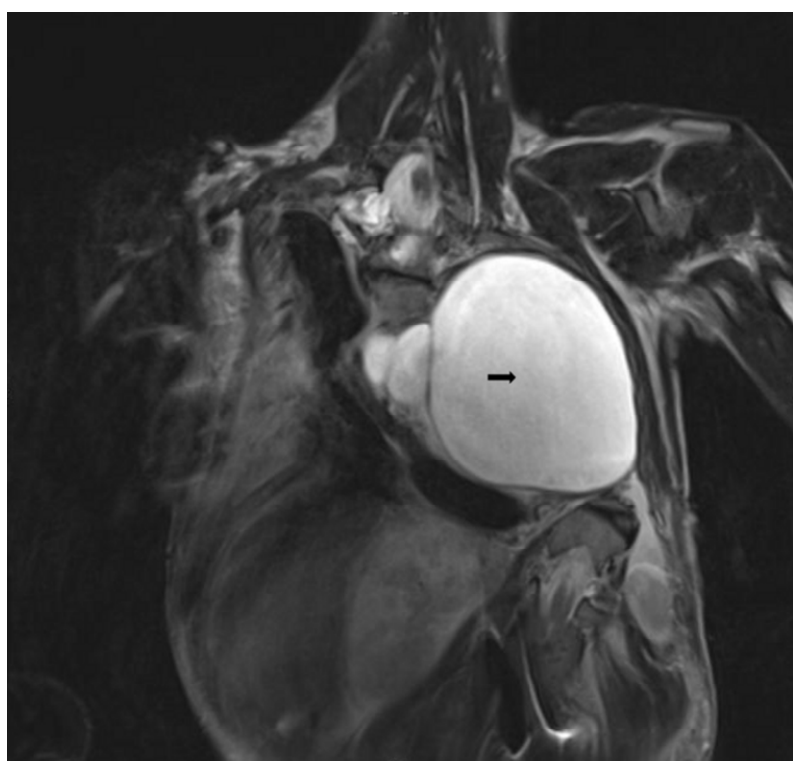

Figure 3. Preoperative coronal MRI demonstrates giant left thoracic meningocele compressing entire left hemithorax.

continue bilevel positive airway pressure (BPAP) and to lie on her left side when supine to relieve meningoceleinduced compression of her right lung.

Upon discharge a week later, the patient was hemodynamically stable and able to move all extremities with good strength. She demonstrated much improved oxygenation, requiring only minimal oxygen on nasal cannula, and was told to continue intermittent BPAP at night. In a follow-up 7 months later, the patient demonstrated continued minimal requirement on nasal cannula, and MRI showed a stable left thoracic giant meningocele (Figures 4 and 5).

\section{Discussion}

Intrathoracic meningoceles are protrusions of dura mater through abnormally enlarged intervertebral foramen or bony defects, and were first reported in 1933 [5]. Anterior spinal meningoceles typically arise from NF-I or Marfan syndrome. NF-I, previously known as von Recklinghausen disease, is an autosomal dominant disorder affecting 1 in 4000 people. Neurofibromas and hyperpigmented areas are classic signs, and spinal deformity is the most common musculoskeletal complication $[4,6,7]$. A posterior mediastinal mass in NF-I patients is most likely to be lateral meningocele [4].

The symptoms associated with intrathoracic meningocele depend on the meningocele's size and relationship with surrounding structures. Small meningoceles can be asymptomatic and incidentally found in a routine chest $\mathrm{x}$-ray. However, larger structures may result in back pain, paraparesis from spinal cord compression, coughing, dyspnea, and palpitation from compression of lung and 


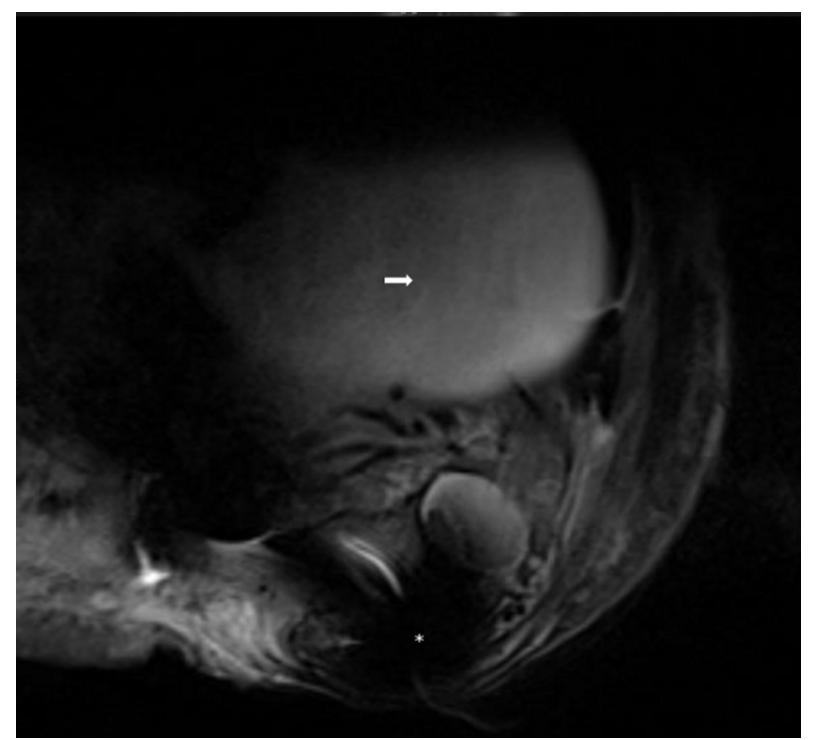

Figure 4. Postoperative axial MRI demonstrates stable left thoracic giant meningocele. Arrow represents stable meningocele, asterisk represents metal construct.

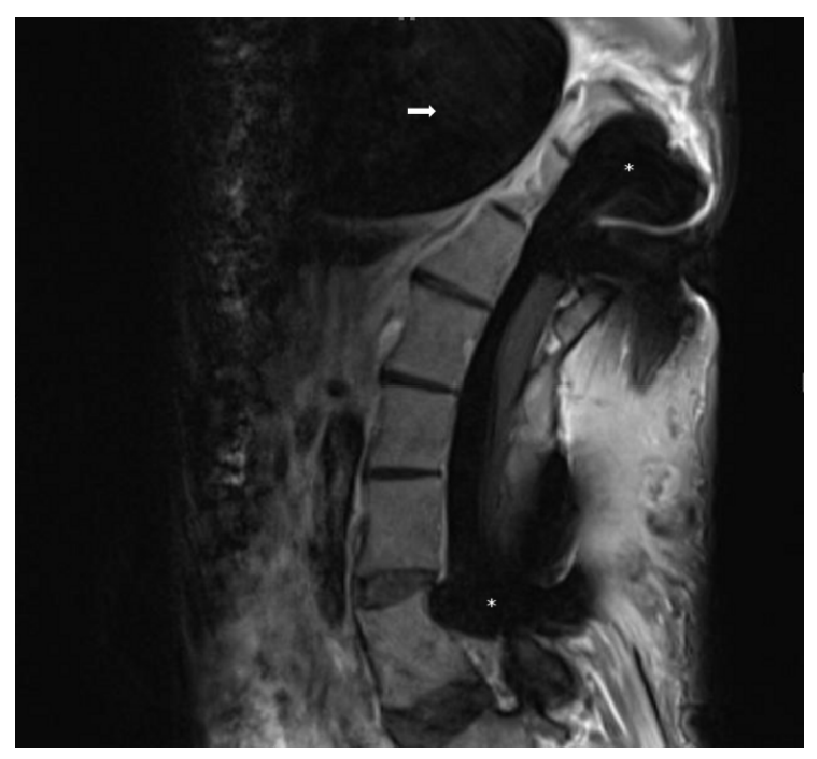

Figure 5. Postoperative sagittal MRI demonstrates stable left thoracic giant meningocele. Arrow represents stable meningocele, asterisks represent metal construct.

mediastinal structures. Meningoceles can also rupture to cause progressive hydrothorax $[3,8]$.

Though meningocele treatment is controversial, lesion excision is widely accepted, especially when the mass is rapidly growing, causing difficulty breathing, or underlying progressive neurological deficit. Laminectomy, thoracotomy, and costotranversectomy are commonly employed surgical techniques $[1,2,4]$, though laminectomy with intradural repair of the cyst is indicated for small and medium-sized meningoceles. Large meningoceles generally require a transthoracic approach for a larger operating space and a lower risk of spinal cord damage [3].

When operative risk is high, shunting is a valuable alternative to the standard surgeries, especially when patients exhibit poor respiratory function [2]. Cystoperitoneal shunts and lumboperitoneal shunts have been shown to be effective methods in draining excess CSF [2,9]. However, providers should be wary of overdrainage, a frequent complication of shunting procedures. Overdrainage, albeit not fatal, can cause chronic headaches, nausea, and vomiting $[2,10]$. Adjustable pressure valves should be used in place of fixed-pressure shunts to allow for noninvasive pressure adjustments [2]. VP shunting was indicated in our case because our patient exhibited high CSF pressure and had a history of unstable respiratory status. In the future, her valve pressure can be adjusted without the need for a revision surgery.

\section{Conclusion}

Ventriculoperitoneal shunting is a method of treating and stabilizing acutely symptomatic giant meningoceles.

\section{REFERENCES}

[1] G. C. de Andrade, O. P. Braga, M. K. Hisatugo, M. A. de Paiva Neto, E. Succi and F. M. Braga, "Giant Intrathoracic Meningoceles Associated with Cutaneous Neurofibromatosis Type I: Case Report,” Arq Neuropsiquiatr, Vol. 61, No. 3A, 2003, pp. 677-681. http://dx.doi.org/10.1590/S0004-282X2003000400029

[2] K. Tanaka, K. Shimizu, S. Kakegawa, K. Oshima and I. Takeyoshi, "Cystoperitoneal Shunt for a Giant Intrathoracic Meningocele under Local Anesthesia," Annals of Thoracic Surgery, Vol. 91, No. 1, 2011, pp. 317-319. http://dx.doi.org/10.1016/j.athoracsur.2010.01.071

[3] A. Y. Oner, M. Uzun, N. Tokgoz and E. T. Tali, "Isolated True Anterior Thoracic Meningocele,” American Journal of Neuroradiology, Vol. 25, No. 10, 2004, pp. 1828-1830.

[4] S. Ebara, Y. Yuzawa, T. Kinoshita, J. Takahashi, I. Nakamura, H. Hirabayashi, J. Kitahara, M. Yamada and K. Takaoka, "A Neurofibromatosis Type 1 Patient with Severe Kyphoscoliosis and Intrathoracic Meningocele,” Journal of Clinical Neuroscience, Vol. 10, No. 2, 2003, pp. 268-272.

http://dx.doi.org/10.1016/S0967-5868(03)00003-1

[5] Y. J. Kim, H. M. Cho, C. S. Yoon, C. K. Lee, T. Y. Lee and J. P. Seok, "Surgical Treatment of Thoracic Menigocele Associated with Neurofibromatosis and Kyphoscoliosis," Korean Society for Thoracic \& Cardiovascular Surgery, Vol. 44, No. 5, 2011, pp. 383-386. http://dx.doi.org/10.5090/kjtcs.2011.44.5.383

[6] E. C. Allibone, R. S. Illingworth and T. Wright, "Neurosis Fibromatosis (von Recklinghausen's Disease) of the Vertebral Column," Archives of Disease in Childhood, Vol. 35, 1960, pp. 153-158.

http://dx.doi.org/10.1136/adc.35.180.153

[7] P. G. Calzavara, A. Carlino, G. P. Anzola and M. P. Pa- 
solini, "Segmental Neurofibromatosis. Case Report and Review of the Literature," Neurofibromatosis, Vol. 1, No. 5-6, 1988, pp. 318-322.

[8] J. Mizuno, H. Nakagawa, T. Yamada and T. Watabe, "Intrathoracic Giant Meningocele Developing Hydrothorax: A Case Report," Journal of Spinal Disorders \& Techniques, Vol. 15, No. 6, 2002, pp. 529-532. http://dx.doi.org/10.1097/00024720-200212000-00018
[9] P. Rukskul, "Lumbo-Peritoneal Shunting Improved Spinal Cord Compression Due to a Large Anterior Sacral Meningocele," Journal of the Medical Association of Thailand, Vol. 88, No. 2, 2005, pp. 265-268.

[10] C. Kondageski, D. Thompson, M. Reynolds and R. D. Hayward, "Experience with the Strata Valve in the Management of Shunt Overdrainage," Journal of Neurosurgery, Vol. 106, No. 2, 2007, pp. 95-102. 